Thorax 1986;41:571-572

\title{
Asthma, eosinophilic pleuropneumonia, and pericarditis without vasculitis
}

\author{
JMM VAN DEN BOSCH, SJ Sc WAGENAAR, CJJ WESTERMANN \\ From the Departments of Pulmonary Diseases and Pathology of St Antonius Hospital, Nieuwegein, \\ The Netherlands
}

The combination of asthma, pulmonary infiltrates, blood eosinophilia, and disease of the pleura or pericardium is rare. Two patients are reported who showed these features, but who lacked evidence of vasculitis.

\section{Case reports}

\section{PATIENT 1}

A 43 year old man had had bronchial asthma with blood eosinophilia, but without evidence of allergy, since 1976 and was treated with oral theophylline and salbutamol and later with inhalations of sodium cromoglycate and beclomethasone. The powdered cromoglycate was replaced by an aqueous solution because of coughing. In 1983 he visited our hospital because of thoracic pain, weight loss, general weakness, and night sweats. Physical examination revealed basal crackles, a pericardial rub, and a temperature of $40^{\circ} \mathrm{C}$. Sputum, stool, urine, and blood examinations for viruses, mycobacteria, bacteria, fungi, and parasites gave negative results. Chest radiography showed progressive bilateral infiltration in the axillary and apical fields and evidence of pericardial effusion. Laboratory investigations gave the following results: white blood cell count $19.7 \times 10^{9} / 1$ with $10.3 \times 10^{9} / 1$ eosinophils; platelet count $824 \times 10^{9} / 1$; erythrocyte sedimentation rate (ESR) $86 \mathrm{~mm}$ in one hour; serum IgE $1.600 \mathrm{kU} / \mathrm{l}$; rheumatoid arthritis latex test positive; rheumatoid arthritis haemagglutinating antibody titre $1 / 640$. There were circulating immune complexes and cryoglobulins. Other serological tests gave negative or normal results.

Open lung biopsy showed hyperplasia of the muscularis mucosae and thickening of the basal membrane of bronchi and bronchioles, with a modest inflammatory reaction containing many eosinophils, but no evidence of obliterative bronchiolitis. The alveoli were found to contain many eosinophils and macrophages. Type II pneumocytes covered the alveolar walls; lymphocytes, plasma cells, and eosinophils were found in the interstitium. No features of vasculitis were seen. The pleural stroma contained locally accumulated plasma cells, lymphocytes, and many eosinophils. The pericardial biopsy showed fibrous thickening with local inflammatory centres of eosinophils and lymphocytes. Similar inflammatory cells were found in pericardial fluid. There were no deposits of immunoglobulins or complement in the vessel walls or along alveolar walls.

Address for reprint requests: Dr JMM Van Den Bosch, St Antonius Hospital, PB 2500, 3430 EM Nieuwegein, The Netherlands.

Accepted 6 December 1985
The patient received $50 \mathrm{mg}$ prednisone daily. After two days a laparotomy was performed because of a perforated diverticulum of the sigmoid colon. Liver and mesenteric biopsies showed no abnormalities. The postoperative course proved uneventful and after a few days both radiological and laboratory abnormalities resolved. Serum, taken before treatment with prednisone, contained no antibodies to cromoglycate; there was no cromoglycate binding activity (Fisons Pharmaceuticals, Loughborough, UK).

\section{PATIENT 2}

A 32 year old woman had had bronchial asthma since 1979 without evidence of allergy and was treated with oral theophylline and salbutamol with inhaled cromoglycate and beclomethasone. In 1981 she was admitted to hospital with acute severe asthma, pericardial effusion, pleurisy, and pulmonary infiltrates affecting the right middle and lower lobes. She complained of fatigue, weight loss, night sweats, and fever. Pericardiocentesis yielded $200 \mathrm{ml}$ of clear fluid, in which $25 \%$ of the cells present were eosinophils. In fluid obtained by bronchoalveolar lavage $48 \%$ of the cells were eosinophils.

Laboratory examination revealed the following: white cell count $15.4 \times 10^{9} / 1$ with $6.6 \times 10^{9} / 1$ eosinophils; platelet count $981 \times 10^{9} / 1$; ESR $105 \mathrm{~mm}$ in one hour. Further extensive serological tests had negative or normal results. Investigations for viruses, mycobacteria, bacteria, fungi, and parasites gave negative results.

Material obtained by open lung biopsy showed local goblet cell metaplasia and a mild eosinophilic inflammatory reaction in bronchi and bronchioli without obliterative bronchiolitis. Alveolar spaces were filled with numerous eosinophils and macrophages, yet the interstitium had hardly changed. There was no evidence of vasculitis. The pleura contained many eosinophils. Immunofluorescence studies of lung tissue showed no abnormal immunological phenomena. Pericardial biopsy showed infiltration by plasma cells, lymphocytes, and a relatively large number of eosinophils.

This patient was treated with $50 \mathrm{mg}$ of prednisone daily. Her clinical progress proved to be good. The test for cromoglycate binding activity in preoperative serum gave a doubtfully positive result owing to cloudiness of the serum.

\section{Discussion}

A viral or bacteriological agent could not be detected and there was no exposure to exotic pathogens. Allergic bronchopulmonary aspergillosis was virtually excluded, as was malignancy and other diseases, such as sarcoidosis, during the follow up of two and four years after the acute episode in 
the two cases. Nitrofurantoin and other drugs are well known causes of pulmonary infiltrates and blood eosinophilia ${ }^{1}$ (PIE syndrome). The combination of pulmonary infiltrates, asthma, blood eosinophilia, and pericarditis has been attributed to the use of cromoglycate. ${ }^{23} \mathrm{~A}$ raised binding activity for cromoglycate, indicating humoral antibodies, has been detected in serum of one patient. ${ }^{24}$ Both of our patients showed low tolerance for cromoglycate, although the binding activity for cromoglycate was not clearly raised. The same combination of symptoms has been described in a patient treated with beclomethasone. ${ }^{5}$

Asthma and cardiac disease may be prominent features of the hypereosinophilic syndrome. ${ }^{6}$ Cardiac disease in this syndrome, however, comprises thromboembolic phenomena, valvular lesions, and abnormalities of endocardium and myocardium. In our patients there was no evidence of such cardiac lesions.

The radiological abnormalities of patient 1 were typical of chronic eosinophilic pneumonia, the so called photographic negative of pulmonary oedema. ${ }^{7}$ Pericarditis has never been reported in chronic eosinophilic pneumonia.

Finally, the combination of asthma, pulmonary infiltrates, blood eosinophilia, and pericarditis or pleurisy has been reported in association with vasculitis by Harkavy. ${ }^{8}$ When extravascular granulomas are also present, the complex of clinical and histological findings fits the description of Churg and Strauss. ${ }^{9}$ We, however, found no signs of vasculitis or granuloma formation in the lung, pericardium, or other biopsy material.

The combination of findings in our patients does not fit into the hitherto accepted classification of PIE syndrome. Several authors have emphasised that the various forms of the PIE syndrome may have an incomplete clinical presentation, may occur in different stages, and may repre- sent a range of manifestations of a single disease process $\stackrel{0}{x}$ rather than separate entities. ${ }^{10}$ Presentation could depend on individual properties, such as asthma or immunological $\stackrel{\mathcal{P}}{\rho}$ state, or even on the causative agent and its route of entry. The pathogenesis of these diseases is largely unknown. An $\bar{\sigma}$ immunological mechanism seems likely; the presence of cir- $\overline{\bar{S}}$ culating immune complexes in our patient 1 seems to support this explanation.

\section{References}

1 Demeter SL, Ahmad M, Tomashefski JF. Drug-induced pulmonary disease. Part I. Patterns of response. Cleve Clin $Q \stackrel{P}{ }$ 1979;46:89-99.

2 Slater EE. Cardiac tamponade and peripheral eosinophilia in a patient receiving cromolyn sodium. Chest 1978;73:878-9.

3 Rosenberg JL, Edlow D, Sneider R. Liver disease and vasculitis in a patient taking cromolyn. Arch Intern Med 1978;138:989-91.

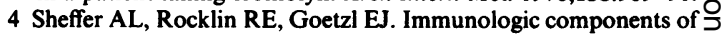
hypersensitivity reactions to cromolyn sodium. $N$ Engl J Med 1975;293:1220-4.

5 Jolobe OMP, Melnick SC. Asthma, pulmonary eosinophilia, and eosinophilic pericarditis. Thorax 1983;38:690-1.

6 Fauci AS. The idiopathic hypereosinophilic syndrome. Clinical, $\vec{\varphi}$ pathophysiologic, and therapeutic considerations. Ann Intern Med 1982;97:78-91.

7 Carrington CB, Addington WW, Goff AM, et al. Chronic eosinophilic pneumonia. $N$ Engl J Med 1969;280:787-98.

8 Harkavy J. Vascular allergy. Pathogenesis of bronchial asthma with recurrent pulmonary infiltrations and eosinophilic polyserositis. Arch Intern Med 1941;78:709-34.

9 Churg J, Strauss L. Allergic granulomatosis, allergic angiitis, and periarteritis nodosa. Am J Pathol 1950;27:277-301.

10 Churg J, Strauss L. Case 46-1980: Interstitial eosinophilic pneumonitis, pleuritis and angiitis. $N$ Engl $J$ Med 1981;304:611. 